\title{
Las competencias en el marco de la convergencia europea: Un nuevo concepto para el diseño de programas educativos
}

\author{
Carolina Fernández-Salinero Miguel \\ Universidad Complutense de Madrid, España
}

\begin{abstract}
Competence is a new concept in "European Space for Higher Education". The concept of competence (professional competence) takes the university to answer work problems, not only to question them. This change needs to understand: What is the meaning of competence? What is its source? What are its different parts? Competence includes elements from education and work, from people and organizations, and from past and present. This paper wants to answer these questions and to make an educational concept useful to work.
\end{abstract}

Key words: Competence, European Space for Higher Education, educational programmes.

\section{RESUMEN}

El Espacio Europeo de Educación Superior introduce un nuevo concepto en el diseño de programas educativos, este concepto es el de competencia. Emanado del ámbito más puramente profesional, el concepto de competencia cambia la orientación de la universidad, tradicionalmente separada del mundo laboral o en paralelo al mismo, y, actualmente, dirigida a dar respuesta a necesidades presentes y futuras que provienen del entorno de trabajo. Este cambio hace necesario un exhaustivo análisis del término competencia, una búsqueda de sus orígenes, una identificación de sus partes fundamentales y una conceptualización que responda, tanto a los intereses universitarios como a los laborales. Este artículo pretende precisamente eso, acotar el término competencia desde la educación y para el trabajo, con referencia a la persona y su organización de destino, sin confundirse con otros términos afines y sin dejar ningún elemento en su configuración.

Descriptores: Competencia, Espacio Europeo de Educación Superior, programas educativos.

\section{RÉSUMÉ}

La Compétence est un nouveau concept introduit dans l'espace européen de l'éducation supérieure pour le design des programmes éducatifs. Émanant de milieu non seulement professionnel, le concept de compétence change l'orientation de l'université, traditionnellement séparée du monde du travail ou parallèle à celui-ci, et actuellement dirigée vers une réponse aux nécessités présentes et futures qui proviennent du monde du travail. Ceci exige une analyse exhaustive du terme compétence, à partir de ses origines, de l'identification de ses composantes fondamentales et une conceptualisation qui réponde et aux intérêts universitaires et à ceux du monde du travail. Ce papier vise précisément ceci : inclure dans la notion de compétence, l'éducation et le travail, le passé et le présent, en référence à la personne et à l'organisation concernée. Et cela sans confondre ce terme avec les autres termes qui lui sont reliés et sans lui enlever aucun élement de sa 
configuration, afin de rendre ce concept utile au but proposé.

Mots-clés: de Tocqueville, le libéralisme, les réseaux de l' éducation, le contrôle de l'enseignement, l'instruction surveillée.

\section{El Espacio Europeo de Educación Superior como marco de referencia}

$\mathrm{E}$ L 25 DE MAYo De 1998 los Ministros de Educación de Francia, Alemania, Italia y Reino Unido acuerdan, en una Declaración solemne aprobada en la Sorbona, impulsar la creación del Espacio Europeo de Educación Superior para promover la movilidad y las posibilidades laborales de los ciudadanos, así como el desarrollo global del continente. Un año más tarde la iniciativa lanzada en París es asumida por los Ministros de Educación de una treintena de países europeos (de dentro y fuera de la Unión), reunidos de nuevo en Bolonia, como una manera de hacer los sistemas de enseñanza superior más compatibles y comparables y favorecer la movilidad en el entorno europeo y la competitividad a escala internacional. Para ello se establecen los siguientes objetivos, con la voluntad de lograrlos antes de la finalización del primer decenio del siglo XXI:

- Adoptar un sistema de títulos fácilmente comprensibles y comparables, por medio, entre otras medidas, de la implantación del suplemento europeo al título (Diploma Supplement), que consiste en un modelo de información unificado, personalizado para el titulado universitario, sobre los estudios cursados, su contexto nacional y las competencias profesionales adquiridas. Tiene como objetivo incrementar la transparencia de las diversas acreditaciones europeas y facilitar su reconocimiento por otras instituciones. El Suplemento Europeo al Título pretende ser un documento comprensivo, abierto a incorporar actualizadamente el aprendizaje a lo largo de la vida, configurando los conocimientos acreditados a una persona por instituciones europeas de enseñanza superior.

- Adoptar una estructura de estudios basada en dos ciclos: grado (estudios de diplomatura y licenciatura) y postgrado (estudios de master y doctorado).

- Establecer un sistema de transferencia y acumulación de créditos (European Credit Transfer System - ECTS) centrado en el alumno, que se base en la carga de trabajo del estudiante necesaria para la consecución de los objetivos de un programa. Estos objetivos se especifican preferiblemente en términos de los resultados del aprendizaje y de las competencias que se han de adquirir (European Comisión, 2005, p.4).

- Promover la movilidad de estudiantes, profesores, investigadores y administradores (capital intelectual de Europa), con la finalidad de fortalecer la dimensión cultural, social, científica y tecnológica de la Unión Europea.

- Promover la cooperación europea en materia de garantías de calidad, por medio del desarrollo de criterios y metodologías comparables (más interactivas, constructivas, interdisciplinares, centradas en el alumno). 
- Promover la dimensión europea en la enseñanza superior para que atraiga a estudiantes de diferentes países, favoreciendo su empleabilidad y competitividad en el mercado laboral internacional.

Iniciativas institucionales y sociales posteriores (Praga, 2001; Salamanca, 2001; Göteborg, 2001; Berlín, 2003; Bergen, 2005), han venido a reafirmar, ampliar y a veces matizar, los objetivos de Bolonia. A su vez, la Unión Europea asumió esta iniciativa desde el objetivo estratégico para la década en curso formulado por el Consejo Europeo de Lisboa en marzo de 2002 de "convertirse en la economía basada en el conocimiento más competitiva y dinámica del mundo, capaz de crecer económicamente de manera sostenible con más y mejores empleos y con mayor cohesión social".

En este marco de adaptación de los estudios y programas al Espacio Europeo de la Educación Superior (EEES), se propone formular los objetivos de enseñanza como resultados de aprendizaje, expresados en términos de competencias vinculadas a los perfiles profesionales para los que preparan los estudios. Emanado del ámbito de la formación y la gestión profesional, el concepto de competencia ha saltado durante los últimos años al terreno de la educación en general (Eurydice, 2002). No hay, sin embargo, consenso acerca de lo que son las competencias y los documentos institucionales no añaden mayor precisión sobre cómo conviene entender este concepto en el trabajo universitario, al margen de su vinculación directa con el mundo laboral. El término no aparece en la declaración de la Sorbona de 1998 y sólo de manera muy genérica en la declaración de Bolonia (1999) y en los comunicados oficiales posteriores. En orden a establecer programas educativos coherentes con este marco parece, pues, aconsejable comenzar clarificando el concepto de competencia, al que todo el mundo se refiere, pero que en contados casos se identifica de manera precisa.

\section{Una aproximación al concepto de competencia}

Cada día es más abundante la investigación y producción bibliográfica relativa al tema de las competencias, a pesar de lo cual existe una gran disparidad de criterios en la manera de asumirlas desde los diferentes ámbitos en que se aplica. Los docentes, por ejemplo, definen las competencias desde su ámbito pedagógico en términos de logros, indicadores de logros, capacidades y conocimientos; los empresarios, en cambio, las abordan desde el ámbito laboral, en términos de desempeño, productividad, eficiencia y profesionalidad ${ }^{2}$. Y, desde un ámbito más teórico, es posible entenderlas en términos de nociones y de conceptos. Como consecuencia, existen grandes dificultades para establecer pautas comunes en orden al diseño de programas basados en competencias, lo cual se convierte en un obstáculo para la continuidad e interacción entre los diferentes niveles y tipos de educación. A continuación se presenta una aproximación al concepto de competencia, apoyándonos para ello en un breve recorrido por diferentes momentos de su desarrollo, una propuesta de definición del término, diferenciación con respecto a otros conceptos similares e identificación de sus características más significativas. 


\section{Fuentes del concepto de competencia}

En el entorno del Espacio Europeo de Educación Superior, la adopción de la idea de competencia se ha visto favorecida por el impulso que le ha dado el proyecto Tuning, cuyos resultados fueron adoptados en nuestro país como esquema de trabajo para el estudio y propuesta de titulaciones en las convocatorias de ANECA (Agencia Nacional de Evaluación de la Calidad y Acreditación). El proyecto Tuning se propuso, en su primera fase, identificar las competencias generales o transversales que debería desarrollar cualquier tipo de estudio superior y las competencias específicas relativas a cada ámbito de conocimiento participante en el proyecto (Administración y Dirección de Empresas, Química, Ciencias de la Educación, Geología, Historia, Matemáticas y Física) (González y Wagenaar, 2003) ${ }^{3}$. La tabla 1 recoge, como primera aproximación al concepto de competencia, algunos de los escenarios históricos más significativos de su desarrollo y su plasmación en la especificación de competencias genéricas de Tuning.

\section{APARICIÓN Y EVOLUCIÓN DEL CONCEPTO DE COMPETENCIA}

Filosofía griega

Competencia instrumental (resolución de problemas)

- La interrogación de la realidad ocupa un papel central al concebirse como apoyo para la resolución de problemas con sentido para las personas.

Competencia interpersonal (capacidad de trabajar en un equipo interdisciplinar)

- Establece relaciones y conexiones entre los diferentes temas y problemas, trascendiendo la mera casualidad (articulación de los saberes de distintas disciplinas).

Competencia sistémica (capacidad para aplicar los conocimientos a la práctica)

- Aristóteles (1999) plantea una continua relación entre el saber y el proceso de desempeño, como puede apreciarse en la Metafisica, en la cual se argumenta que todos los hombres tienen las mismas facultades (capacidades para el conocimiento) y lo que los hace diferentes es el uso que le dan a dichas facultades, así como que las personas poseen por naturaleza el deseo de saber, el cual irrumpe por el placer y su utilidad.

Competencia sistémica (capacidad de aprender)

- Platón (1972) muestra en La República cómo el ser humano puede caer en el error de asumir la realidad desde la apariencia. En la alegoría de la caverna propone que para llegar al verdadero conocimiento se requiere de una búsqueda constante de la esencia de las cosas, trascendiendo lo aparente y superando los errores de la percepción. Esto es posible debido a que toda persona posee en su alma la capacidad de aprender.

\section{Lingüística}

Competencia instrumental (competencia lingüistica)

- Noam Chomsky introduce el concepto de competencia en 1965, dentro de su teoría de la gramática generativa transformacional y bajo la denominación de competencia lingüistica, la cual se refiere al conocimiento de las reglas o principios abstractos que regulan el sistema lingüístico, conocimiento que se evidencia en las actuaciones y desempeños lingüísticos individuales.

Competencia instrumental (competencia comunicativa)

- Hymes (1980) establece el concepto de competencia comunicativa, con la que plantea los usos y actos concretos dados a partir del lenguaje, dentro de contextos específicos. En este sentido, una persona competente en el lenguaje es aquella que lo emplea en un contexto de relación con los otros entendiendo y haciéndose entender. 


\section{Filosofía moderna}

\section{Competencia interpersonal (competencia interactiva)}

- Jurgen Habermas (1987) menciona el concepto de competencia bajo dos términos: Competencia comunicativa y competencia interactiva. Ambas se refieren al uso del lenguaje por sujetos considerados hablantes-oyentes que emplean el lenguaje para entenderse acerca de un determinado tema, produciéndose el significado dentro del mismo ámbito del uso. Habermas considera que las competencias tienen unos componentes universales que permiten el entendimiento entre las personas.

\section{Sociología}

Competencia interpersonal (competencia ideológica)

- Eliseo Verón propone, en los años 1969 y 1970, el concepto de competencia ideológica, definido como el conjunto de maneras específicas de establecer selecciones y organizaciones de un determinado discurso. Las personas realizan selecciones de palabras y las ponen en un determinado orden acorde con ciertas reglas. La competencia ideológica tiene también carácter generativo.

\section{Mundo laboral}

Competencia sistémica (capacidad de aprendizaje permanente)

- Según Hyland (1994), las competencias surgieron en este ámbito en la década de los años sesenta, cuando se comenzaron a implementar nuevos procesos de organización del trabajo como consecuencia de la necesidad empresarial de promover el aprendizaje organizacional y la movilidad laboral (Mertens, 2000). En la década de los 80 se da un gran impulso a la mejora de las condiciones productivas y toma auge la formación de los recursos humanos con parámetros de eficiencia y eficacia. Aparece entonces el enfoque de las competencias laborales, sustentado en cuatro ejes: Identificación, normalización, formación y certificación (Tobón, 2004).

Competencia sistémica (creatividad y liderazgo) y competencia interpersonal (capacidad de trabajar en equipo)

- Durante la década de los noventa se consolida la gestión del talento humano basada en las competencias, construyéndose metodologías específicas para llevar a cabo los procesos de selección, capacitación, remuneración, ascenso y evaluación. Este enfoque premia más el saber hacer que el mero conocimiento, se promueve la formación continua de la plantilla, flexibilidad laboral para asumir el cambio, alta capacidad para trabajar en equipo, liderazgo y creatividad, etc.

\section{Psicología cognitiva}

Competencia instrumental (competencias cognitivas)

- Una de las aportaciones más actuales es la de las denominadas competencias cognitivas, referidas a procesos mediante los cuales se procesa la información acorde con las demandas del entorno, poniéndose en acción esquemas cognitivos, técnicas y estrategias, lo que permite al sujeto percibir, conocer, explicar, comprender e interpretar la realidad (Tobón, 2004). Otras aportaciones significativas que podemos señalar son las que se apoyan en las siguientes tres líneas de investigación: Teoría de la modificabilidad estructural cognitiva (posibilidad de modificación de las competencias por experiencias de aprendizaje), teoría de las inteligencias múltiples de Gardner (1987, 1988, 1993) (que distingue ocho tipos distintos) y enseñanza para la comprensión (que se apoya en el aprendizaje abierto y contextualizado). 


\section{Psicología laboral \\ Competencia instrumental (competencias de umbral) y competencia interpersonal y sistémica (competencias diferenciadoras)}

- El concepto de competencia fue implementado en este ámbito por McClelland en los años setenta (Spencer et al, 1994), a partir de una serie de estudios en los cuales encontró que las tradicionales pruebas de desempeño no eran efectivas para predecir el éxito en el trabajo.

Por ello, este autor propuso tener más en cuenta las características de los trabajadores y sus comportamientos concretos ante situaciones laborales. Una aportación interesante es también el concepto de competencias de umbraly competencias diferenciadoras (Gallego, 2000). Las primeras permiten el desempeño normal o adecuado de una actividad; las segundas se refieren a características que posibilitan a las personas desempeñar de manera sobresaliente una actividad, aportándole ventajas competitivas a la organización en su conjunto.

\section{Pedagogía}

Competencia instrumental (capacidad de análisis y sintesis, de resolución de problemas y toma de decisiones)

- Desde un punto de vista pedagógico, el tema se comienza a abordar en la década de los años noventa, en el marco del establecimiento de metodologías innovadoras para evaluar los aprendizajes y la calidad de la educación (Jurado, 2003). Se buscaba con ello superar las metodologías tradicionales basadas en la memorización, la acumulación y la repetición mecánica de datos, para privilegiar los procesos cognitivos (percepción, atención, comprensión, inteligencia y lenguaje), las habilidades cognitivas (interpretación, argumentación y proposición) y la resolución de problemas con sentido para los estudiantes.

Competencias básicas (fundamentales para poder adquirir las siguientes y más específicas)

- Las aportaciones de la lingüística, junto con la influencia de la teoría del procesamiento de la información, las inteligencias múltiples y las competencias laborales, están consolidando poco a poco y, cada vez más, el concepto de competencias básicas (competencias comunicativas, matemáticas, sociales, en ciencias naturales, técnicas, etc.), dentro de los diseños curriculares.

Tabla 1. Escenarios históricos del concepto de competencia (elaboración propia).

\section{Qué entendemos por competencia}

Una vez realizado el recorrido precedente por algunos de los hitos que han ido configurando el concepto de competencia y que nos permiten comprobar la interdisciplinariedad con que se ha ido conformando, parece necesario esclarecer a continuación ese concepto en el momento actual.

Mucho se ha escrito sobre las competencias, pero todavía existen grandes vacíos y desacuerdos frente a su definición. Esto constituye un serio obstáculo para el diseño de programas educativos que tengan como soporte un enfoque basado en las mismas. Asimismo, las definiciones que cotidianamente se construyen están determinadas por la manera en que se identifican los aspectos tangibles e intangibles relacionados con ellas. Por ejemplo, con frecuencia se entienden las competencias sociales (que tienden a ser intangibles) de manera diferente a las competencias técnicas (que tienden a ser más tangibles). Y, por otro lado, el significado del concepto varía entre los países, refiriéndose indistintamente a títulos educativos, categorías laborales, puestos de 
trabajo, etc. El término competencia es, por tanto, un concepto confuso, ya que no está claro si la competencia se refiere a lo que las personas son capaces de hacer, deben ser capaces de hacer, tienen que hacer o realmente hacen para alcanzar el éxito profesional.

Para comenzar a perfilar una definición, podemos partir de su origen etimológico. Lo primero que hay que apuntar al respecto es que el significante competencia es antiquísimo. En castellano existen dos términos: competer y competir, los cuales provienen del verbo latino competere, que significa ir una cosa al encuentro de otra, encontrarse, coincidir. A partir del siglo XV competer adquiere el significado de pertenecer a, incumbir, corresponder a. De esta manera, se constituye el sustantivo competencia y el adjetivo competente, que quiere decir apto o adecuado. A partir del mismo siglo XV competir se usa con el significado de pugnar con, rivalizar con, contender con, dando lugar a los sustantivos competición, competencia, competidor, competitividad, así como al adjetivo competitivo (Corominas, 1987 y Corripio, 1984).

La etimología deja todavía demasiado campo abierto, que nos obliga a buscar un marco teórico en el que situar este concepto. El recorrido que realizamos anteriormente nos muestra que, más que en las fuentes disciplinares de donde proviene (lingüística, filosofía, sociología, psicología cognitiva y laboral, etc.), conviene situar ese marco en el perfil interdisciplinar de esta idea.

Un planteamiento teórico bastante actual y que nos puede resultar útil para comprender mejor este concepto procede del pensamiento complejo, concebido como un método de construcción del conocimiento que se basa en el tejido de relaciones entre las partes y el todo desde la continua organización-orden-desorden (Morín, 1996). Asumir la complejidad como epistemología de las competencias implica reconocerlas como un referente inacabado y en constante construcción-deconstrucciónreconstrucción, requiriéndose continuamente del análisis crítico y la autorreflexión para comprenderlo y usarlo. De aquí que construir el concepto de competencia desde la complejidad, más que dar cuenta de una "realidad objetiva" y precisa, es elaborar la lógica de las relaciones conceptuales que nos permita entenderlo en un marco sociohistórico.

El concepto de competencia debe ser asumido, desde esta perspectiva, como un saber hacer razonado para enfrentarse a la incertidumbre. De esta manera, las competencias no podrían abordarse únicamente como comportamientos observables, sino como una compleja estructura de atributos necesarios para el desempeño en situaciones diversas, donde se combinan conocimientos (tácitos y explícitos) ${ }^{4}$, habilidades, actitudes y valores, con tareas que se tienen que desempeñar en determinadas situaciones.

Un enfoque de tales características nos debe permitir diferenciar el concepto de competencia de otros conceptos afines, que si bien guardan relación con él, no son equivalentes, como es el caso de las aptitudes y los rasgos de personalidad. Estos últimos permiten caracterizar a los individuos y explicar la variación de sus comportamientos en la ejecución de tareas específicas, mientras que las competencias afectan a la puesta en práctica integrada de aptitudes, rasgos de personalidad y también conocimientos adquiridos para cumplir bien una tarea compleja en un contexto determinado y dentro del espíritu de una estrategia y cultura identificadas (Levy-Leboyer, 1997). No se puede 
decir pues que las competencias no estén en relación con las aptitudes y los rasgos de personalidad, pero constituyen una categoría específica de características individuales que tienen lazos estrechos con los valores y con los conocimientos adquiridos. Tienen además un carácter "local", es decir, dependen del marco de referencia en el que son elaboradas, aprendidas o desarrolladas y, en consecuencia, utilizadas.

Algunos otros términos relacionados con el de competencia, pero que no deben confundirse con él, serían los siguientes (Tobón, 2004):

- Cualificaciones profesionales: término que se ha empleado para referirse a la capacidad general de desempeñar todo un conjunto de tareas y actividades relacionadas con un oficio y apoyadas en una certificación acreditativa. Las competencias a diferencia de las cualificaciones, enfatizan su desempeño en procesos personales y profesionales específicos, donde lo central es la adaptación al cambio y la flexibilidad en el desempeño de actividades. Las cualificaciones profesionales serían por tanto un elemento previo para la definición de las competencias.

- Capacidades. son condiciones cognitivas, afectivas y psicomotrices fundamentales para aprender y denotan la dedicación a una tarea. Son el desarrollo de las aptitudes. Las competencias, en este sentido, tienen como uno de sus componentes las capacidades con el fin de llevar a cabo una actividad.

- Destrezas: son mediadoras entre las capacidades y las habilidades y su adquisición supone el dominio, tanto de la percepción frente a los estímulos como de la reacción eficaz para ejecutar la tarea. La eficacia y la flexibilidad son dos cualidades que las definen (Sánchez Cerezo, 1991, p.147). Las competencias tienen las destrezas como base para la actuación, pero difieren de éstas en que integran el conocimiento, los procedimientos y las actitudes en la búsqueda de objetivos a corto y largo plazo.

- Habilidades: consisten en cualidades que permiten realizar tareas y actividades con eficacia y eficiencia. Las competencias buscan también la eficiencia y la eficacia, pero además integran la comprensión de la situación, conciencia crítica, espíritu de reto, responsabilidad por las acciones y desempeño basado en indicadores de calidad.

- Actitudes: son disposiciones afectivas para la acción y constituyen el motor que impulsa al comportamiento en los seres humanos. Inducen igualmente a la toma de decisiones y a desplegar un determinado tipo de comportamiento acorde con las circunstancias del momento. A este respecto, las competencias se componen de cuatro saberes: saber conocer, saber hacer, saber estar y saber ser. El saber ser, a la vez, está integrado por valores, estrategias psicoafectivas y actitudes. Por lo tanto, las competencias son un proceso de actuación amplio y las actitudes uno de sus componentes.

A partir de estas ideas, se propone conceptuar las competencias como estructuras complejas de procesos que las personas ponen en acción-actuación-creación para resolver problemas y realizar actividades (de la vida cotidiana y del contexto laboral- 
profesional), orientadas a la construcción y transformación de la realidad. Integran el saber conocer (observar, analizar, comprender y explicar), el saber hacer (desempeño basado en procedimientos y estrategias), el saber estar (participación y trabajo colaborador) y el saber ser (automotivación, iniciativa, liderazgo y creatividad), teniendo en cuenta los requerimientos específicos del entorno, las necesidades personales y las condiciones de incertidumbre, con autonomía intelectual, conciencia crítica y espíritu de reto, asumiendo al mismo tiempo las consecuencias de sus actos y buscando el bienestar humano. Las competencias, en definitiva, están constituidas por procesos subyacentes (cognitivo-afectivos), así como también por procesos públicos y demostrables, que permitan elaborar algo de sí para los demás con rigurosidad (Gallego, 1999).

El concepto de competencia así conformado, implica la capacidad de movilizar varios recursos cognitivos para hacer frente a un tipo de situaciones y es indisociable de la noción de desarrollo (Levy-Leboyer, 1997). Esta delimitación insiste en cuatro aspectos, según Perrenoud (2004, p.11):

- Las competencias no son en sí mismas conocimientos, habilidades o actitudes, aunque movilizan, integran, orquestan tales recursos.

- Esta movilización sólo resulta pertinente en una situación y cada situación es única, aunque se pueda tratar por analogía con otras ya conocidas.

- El ejercicio de la competencia pasa por operaciones mentales complejas, sostenidas por esquemas de pensamiento, los cuales permiten determinar (más o menos de un modo consciente y rápido) y realizar (más o menos de un modo eficaz) una acción relativamente adaptada a una situación.

- Las competencias se desarrollan mediante procesos de formación, pero también a merced de la navegación cotidiana del practicante, de una situación a otra (Le Boterf, 2001).

La descripción de una competencia en tales términos debe tener como base unos componentes estructurales específicos. Estos componentes se han retomado del enfoque de las competencias laborales, adaptándolo en lenguaje y finalidades al campo de la educación y concibiéndolo además desde una perspectiva más amplia de desarrollo humano, según plasma la tabla 2.

1. Identificación de la competencia: nombre y descripción de la competencia mediante un verbo en infinitivo, un objeto sobre el cual recae la acción y una condición de calidad. Permite elaborar mapas de competencias interrelacionadas y asociadas a diferentes desempeños educativos y profesionales.

2. Elementos de competencia: desempeños específicos que componen la competencia identificada.

3. Criterios de desempeño: resultados que una persona debe demostrar en situaciones reales de la vida individual, social y profesional, teniendo como base unos determinados requisitos de calidad para que el desempeño sea idóneo. Criterios y elementos conforman las unidades de competencia. 
4. Saberes esenciales: conocimientos requeridos para que la persona pueda lograr los resultados descritos en cada uno de los criterios de desempeño, los cuales se clasifican en saber conocer, saber hacer, saber estar y saber ser.

5. Rango de aplicación: diferentes clases, tipos y condiciones en las que se aplican los elementos de competencia y los criterios de desempeño, lo cual tiene como requisito que tales condiciones impliquen variantes en la competencia.

6. Evidencias requeridas: pruebas necesarias para juzgar y evaluar las competencias de una persona, acordes con los criterios de desempeño, los saberes esenciales y el rango de aplicación de las competencias.

7. Problemas: dificultades que la persona debe resolver de manera adecuada mediante la competencia.

8. Caos e incertidumbre: descripción de las situaciones de incertidumbre asociadas generalmente al desempeño de las competencias, las cuales deben ser afrontadas mediante estrategias.

Tabla 2. Componentes estructurales de una competencia (adaptación de Tobón, 2004, p.49).

\section{Características del concepto de competencia}

El análisis realizado del concepto de competencia nos permite identificar sus características más relevantes, que podemos resumir en las siguientes:

- Aplicación: las competencias se manifiestan en desempeños contextualizados en torno a la resolución de problemas. Son esencialmente un tipo de conocimiento o habilidad ligado a ciertos desempeños que van más allá de la memorización o la rutina. Se trata de un conocimiento o habilidad derivado de un aprendizaje significativo. Supone igualmente el uso creativo de los conocimientos y habilidades adquiridos.

- Carácter dinámico: al entender que las competencias no se agotan en un momento dado, sino que su dominio suele tener una progresión a lo largo de la vida (Sarramona et al 2005, p.203).

- Carácter transversal: las competencias se deben lograr mediante la actuación coordinada de diversas materias y profesores. Ello es fácilmente explicable si se tiene en cuenta que son de naturaleza eminentemente aplicativa, para resolver problemas y situaciones, los cuales raramente son abarcables desde una única disciplina académica (Sarramona et al, 2005, p.203).

- Complejidad: las competencias no consisten en la mera adquisición y aplicación de las capacidades cognoscitivas, afectivas y psicomotrices de manera asilada. Constituyen más bien la integración de estos componentes en estructuras complejas que implican la consecución de una finalidad determinada, dentro de los diferentes ámbitos de la vida individual, social y profesional. Toda competencia expresa, en realidad, una síntesis del desempeño de una persona en sus espacios vitales.

- Contextualización: las competencias se forman en interacción con los contextos. Éstos últimos requieren demandarlas y posibilitar todos los recursos necesarios para su obtención, ya que de lo contrario, las personas no sentirán la necesidad 
de adquirirlas o no poseerán los recursos para hacerlo. Por su parte, las personas, al construir las competencias desde su propia perspectiva, cambian los entornos. Por lo tanto, los contextos actúan sobre las personas y las personas actúan sobre los contextos, estableciéndose así una interdependencia mutua.

- Idoneidad: es la capacidad de actuar en relación con unos determinados requerimientos, por ejemplo profesionales, que se traducen en normas o estándares establecidos. $\mathrm{O}$, lo que es lo mismo, se trata de la capacidad de realizar las actividades que demanda la situación conforme a unos niveles de desempeño esperados.

- Integración: las competencias coordinan intenciones (interés por hacer las cosas mejor o por hacer algo original), acciones (fijación de objetivos, responsabilidad sobre los resultados, asunción de riesgos calculados) y resultados (mejora en la calidad, en la productividad, en innovaciones en servicios y productos) (Tobón y Fernández Tobón, 2002, p.55).

- Interdependencia: no es posible pensar en competencias puras, pues todas ellas se entrelazan compartiendo conocimientos, habilidades y actitudes.

- Procesamiento de la información: las competencias constituyen una manera de organizar la información, posibilitando a quienes las poseen llevar a cabo una actividad o un trabajo, así como resolver un problema particular.

- Resolución de problemas: en este punto ha habido dos tendencias fundamentales. Una se centra en la utilización contextual de estrategias y procedimientos (Pozo, 1994), la otra apunta a resolver problemas aplicando habilidades generales (Nickerson et al, 1994). Las dos perspectivas tienen implicaciones pedagógicas. En la primera se enseña a resolver problemas en un contexto específico y, en la segunda en cambio, se enseña a resolver problemas con aplicación en múltiples contextos.

- Transferencia: las personas poseen competencias en el momento en el cual pueden transferir los conocimientos, habilidades y actitudes a la resolución de nuevos problemas.

\section{Propuesta de clasificación de competencias}

El concepto de competencia se presenta, en virtud de lo expuesto hasta este momento, como la combinación de atributos que atañen a diversos órdenes de la persona relacionados con: a) los conocimientos, aptitudes y destrezas técnicas (saber); b) las formas metodológicas de proceder en una actividad (saber hacer); c) las pautas y formas de comportamiento individuales y colectivas (saber estar); d) las formas de organización e interacción (saber ser). Existen infinidad de clasificaciones. Así, por ejemplo, en el ámbito del trabajo las competencias pueden clasificarse en laborales y profesionales. Las primeras son propias de obreros cualificados, se desarrollan mediante estudios técnicos de formación profesional y se aplican a actividades muy específicas; las segundas son exclusivas de profesionales que han realizado estudios de educación superior y se caracterizan por su alta flexibilidad y amplitud, así como por la capacidad para abordar imprevistos y para afrontar problemas de alto nivel de complejidad (Tobón, 2004). 
Algunos analistas, como Punk (1994), llegan a distinguir hasta cuatro tipos de competencias, exigidas todas ellas por las rápidas transformaciones económicas y sociales. La adaptación de estas categorías, realizada en nuestro país por Echeverría y sus colaboradores (1999), indica que un profesional necesita: competencia técnica (conocimientos y destrezas requeridos para abordar tareas profesionales en un amplio entorno laboral); competencia metodológica (análisis y resolución de problemas, al mismo tiempo que transferencia de soluciones a otros contextos); competencia participativa (colaboración en el trabajo y trabajo en equipo); competencia personal (participación activa en el trabajo, toma de decisiones y aceptación de responsabilidades).

A partir de algunas clasificaciones existentes, como las ya señaladas, y con la intención de establecer una guía útil para el diseño posterior de programas educativos, podemos proponer la siguiente ordenación de competencias que orientan perfiles profesionales. Nos vamos a basar para ello en los ejes de amplitud y participantes.

\section{I) Por razón de su amplitud:}

Un primer eje a la hora de clasificar las competencias es el continuo generalidadespecificidad, en función del mismo podemos distinguir tres tipos de competencias.

\section{a) Competencias clave o competencias básicas.}

Son aquellas competencias fundamentales para desenvolverse con eficacia en diversos contextos sociales y laborales (OECD, 2002). Según el Grupo de Trabajo B de la Comisión Europea (2004, p.7) "representan un paquete multifuncional y transferible de conocimientos, destrezas y actitudes que todos los individuos necesitan para su realización personal, inclusión y empleo. Éstas deberían haber sido desarrolladas al finalizar la enseñanza obligatoria y deberían actuar como la base sobre la cual se forman los demás tipos de competencias, dentro de un aprendizaje a lo largo y ancho de la vida". Cabe adscribir en este grupo las denominadas competencias cognitivas de procesamiento de información, que incluyen tres competencias clave: interpretativa (se fundamenta en la comprensión de la información, buscando su sentido), argumentativa (se sustenta en el conjunto de conocimientos, habilidades y actitudes dirigidas a la explicación de determinados procesos) y propositiva (consiste en proponer hipótesis para explicar ciertos hechos o construir soluciones a problemas) (Tobón, 2004).

\section{b) Competencias genéricas o competencias transversales.}

Son aquellas competencias comunes a varias ocupaciones o profesiones, que se relacionan con la puesta en práctica integrada de aptitudes, rasgos de personalidad, conocimientos adquiridos y también valores. Sin ser específicas de un determinado puesto de trabajo o profesión, son necesarias para desempeñar de manera adecuada el nivel requerido por el empleo, al tiempo que permiten una continua adaptación al cambiante mundo laboral. Se caracterizan porque aumentan las posibilidades de empleo, al favorecer en las personas el cambio de un trabajo a otro; favorecen la gestión, consecución y conservación del empleo; permiten la adaptación a diferentes entornos laborales; no están ligadas a una ocupación en particular; se consiguen mediante 
procesos sistemáticos de enseñanza y aprendizaje; su adquisición y desempeño puede evaluarse de manera rigurosa.

c) Competencias técnicas o competencias especificas.

Son aquellas competencias propias de una determinada ocupación o profesión. Tienen un alto grado de especialización y se adquieren a través de procesos específicos, generalmente llevados a cabo en programas de formación profesional o educación superior, fundamentados en conocimientos científicos y en técnicas compartidas y comunicables. Se clasifican a su vez en: fundamentales, obligatorias, optativas y adicionales. Las competencias fundamentales son comunes a todo el campo ocupacional o profesional y se requieren como apoyo para las demás competencias. Las competencias obligatorias son aquellas comunes a los puestos de trabajo de una determinada ocupación o familia profesional (son indispensables para obtener la titulación). Las competencias optativas, por su parte, son específicas de un grupo de puestos de trabajo dentro de la ocupación o profesión (para lograr la titulación se requiere competencia en una o en varias). Por último, las competencias adicionales se corresponden con funciones muy especializadas que desempeñan menos del $20 \%$ de las personas que trabajan en una ocupación o profesión y no son necesarias para lograr una titulación (Tobón, 2004).

II) Por razón de los participantes:

En razón de los sujetos implicados, podemos hablar de competencias individuales o personales y competencias colectivas u organizativas.

a) Competencias individuales o personales.

Estas competencias incluyen las básicas, genéricas y específicas, con la única salvedad que harían referencia a éstas en diferentes condiciones y con peculiaridades concretas en función de cada una de las personas que se ven implicadas en su adquisición y ejecución.

Zabalza (2003) identifica cuatro grandes grupos de competencias personales, denominadas "competencias de tercer nivel" por Aubrun y Orifiamma (1990, pp.2123) y consideradas como: competencias referidas a comportamientos profesionales y sociales (actuaciones técnicas junto con otras de gestión, toma de decisiones o trabajo compartido), competencias referidas a actitudes (motivación personal, compromiso, maneras de tratar a los demás y capacidad de adaptación), competencias referidas a capacidades creativas (asunción de riesgos, originalidad, búsqueda de soluciones nuevas) y competencias de actitudes existenciales y éticas (autocrítica, proyecto personal, valores humanísticos y compromiso social y ético). A su vez, Van Vucht Tijssen y De Weert (2005) mantienen una distinción que se sustenta en cuatro áreas fundamentales: competencias específicas (requisitos cognitivos individuales para el desarrollo profesional), competencias genéricas (competencias transversales, adecuadas para diferentes profesiones), competencias reflexivas (juicio crítico y conocimiento intuitivo) y competencias académicas (aquellas que incluyen tanto aspectos profesionales o productos a conseguir, como hábitos intelectuales o procesos a desarrollar, y que se refieren tanto a los alumnos en formación como a los profesores responsables del proceso de enseñanza- 
aprendizaje). Y como complemento a todo ello, Iriarte (2005) advierte de la existencia de seis competencias personales tradicionales (especialización, aprendizaje a lo largo de toda la vida, confianza y colaboración, creatividad, ensamblaje y autogestión), a las que añade una nueva denominada la séptima competencia: "La gestión de la complejidad". Esta competencia tiene cuatro rasgos distintivos, la bipolaridad como método (capacidad de análisis desde diferentes perspectivas), la lógica de la colaboración y la autonomía, la interconexión entre conocimiento y acción y entre lo particular y lo colectivo, así como el efecto mariposa (acciones individuales pueden tener efectos multiplicadores).

\section{b) Competencias colectivas o competencias organizativas.}

La competencia colectiva es una preocupación creciente de las empresas y organizaciones, pero todavía no está del todo bien identificada. Podemos adelantar que la competencia colectiva es un resultado. Aparece a través de la cooperación y de la sinergia existente entre las competencias individuales. Suele ser pertinente buscarla a partir de cierto número de características de funcionamiento de una unidad o grupo.

En un proyecto o proceso grupal se reconocerá que existe una competencia colectiva cuando se manifieste (Le Boterf, 2001, p.139):

- Una elaboración de representaciones compartidas de un problema operativo, de un objetivo a alcanzar, de un esquema común de interpretación.

- Una comunicación eficaz: existencia de un lenguaje operativo común, capacidad de reacción ante señales débiles, adaptación de los lenguajes a situaciones vividas colectivamente (de rutina, urgencia, inéditas).

- Una cooperación eficiente entre los miembros del equipo: capacidad de negociar los conflictos con frialdad, visibilidad de la contribución que se espera de cada uno en la actuación colectiva, elección de modos de cooperación apropiados para las diversas formas de organización.

- Un saber aprender colectivamente de la experiencia: la formalización y la utilización de resultados, la existencia y el funcionamiento de procesos de capitalización de las prácticas profesionales, el funcionamiento de grupos de intercambio de prácticas.

La competencia colectiva busca la complementariedad y el encadenamiento de competencias. La puesta en práctica de las competencias de cada actor está relacionada con la competencia de los demás actores de la cadena. Sólo en la medida en que cada individuo encuentra competencias complementarias a las suyas, podrá poner estas últimas plenamente en práctica. No obstante, pueden existir núcleos comunes de competencias entre varias ocupaciones o profesiones. Es uno de los componentes posibles de este tipo de competencia. 


\section{Elementos para el diseño de programas basados en competencias}

Tras los análisis conceptuales anteriores, podemos perfilar ahora algunos elementos para el diseño de programas de formación competencial en el marco del EEES, siempre teniendo en cuenta que un programa de formación es un proyecto de actuación en el que, a partir de la detección de unas necesidades concretas, se realiza una previsión sistemática de objetivos, que en este caso se plantearán en términos de competencias, se definen estrategias para su consecución, seleccionándose los medios y recursos necesarios, y se especifican criterios de evaluación, rigurosos y realistas. Estamos, por lo tanto, dentro de una estructura general de planificación de la formación (FernándezSalinero, 2004).

\section{Niveles en la adquisición de competencias}

Si la identificación de competencias es un paso esencial en la adaptación de las titulaciones y programas al EEES, no hay que olvidar que, en la realidad, la competencia no existe por sí misma, independientemente de un sujeto que la posee y del que no puede disociarse. Lo que realmente existe son personas más o menos competentes. Por lo tanto, resulta importante precisar qué es lo que puede entenderse por persona competente ¿Cómo se reconocerá a una persona competente? ¿Qué es lo que permitirá calificar a una persona de competente en un determinado dominio? De acuerdo con Le Boterf (2001, pp.123-127), podemos distinguir tres niveles básicos de adquisición de competencias, en cada uno de los cuales cabe identificar los cuatro componentes a los que nos hemos venido refiriendo:

- Saber conocer: incluye los metaconocimientos y la contextualización del saber.

- Saber hacer: incluye la gestión/modo de intervención y la gestión del tiempo.

- Saber estar: incluye el grado de autonomía y la fiabilidad.

- Saber ser incluye la imagen propia.

El nivel de principiante. Sus características fundamentales son las siguientes:

- Metaconocimientos: conoce mal sus potencialidades, organiza sus conocimientos en función del enunciado del problema, tiene un saber fragmentario, se queda en la estructura superficial de los problemas y tiene poca capacidad para utilizar sus conocimientos en los momentos oportunos.

- Contextualización del saber: conocimientos generales y poco contextualizados.

- Gestión/modo de intervención: visión parcial de una situación, dificultad para distinguir lo esencial de lo accesorio, mala selección de las informaciones, progresión paso a paso y analítica, recurre poco o nada a la intuición, hace mucha referencia a reglas y procedimientos generales.

- Gestión del tiempo: en aprendizaje.

- Grado de autonomía: poca autonomía, aplicación sujeta a reglas formales.

- Fiabilidad: poca fiabilidad, pero con éxitos puntuales.

- Imagen propia: incierta, en plena construcción y en busca de un modelo. 
El nivel de maestría profesional. Sus características son:

- Metaconocimientos: buen conocimiento de su saber, capacidades y competencias.

- Contextualización del saber: contextualizado.

- Gestión/modo de intervención: estrategias de estudio y de solución de problemas, sabe tomar decisiones razonadas, visión global y coherente de las situaciones, confianza limitada en la intuición, adaptación de las reglas a un contexto.

- Gestión del tiempo: la domina.

- Grado de autonomía: sabe interpretar las reglas, simplifica o abandona progresivamente las reglas de formación, es autónomo.

- Fiabilidad: fiable.

- Imagen propia: confianza en sus capacidades y en los conocimientos adquiridos.

El nivel de experto. Se caracteriza por:

- Metaconocimientos: organiza sus conocimientos a partir de esquemas y en bloques, conoce los múltiples usos de los recursos, tiene grandes automatismos y saber hacer incorporados, capacidad para aprovechar el momento adecuado para poner en práctica sus conocimientos.

- Contextualización del saber. muy contextualizado.

- Gestión/modo de intervención: reacciona de manera intuitiva y global, no recurre a los procedimientos habituales, visión sintética, separa lo esencial de lo accesorio, se anticipa a partir de señales débiles.

- Gestión del tiempo: actúa rápidamente pero sabe elegir el ritmo adecuado, reacciona y se anticipa, tiene rapidez de ejecución y gran soltura.

- Grado de autonomía: total, puede fijar sus límites, sabe dar nuevas interpretaciones a las reglas, tiene capacidad para crear sus propias reglas.

- Fiabilidad: una regularidad muy grande de la competencia.

- Imagen propia: muy congruente, conoce los puntos fuertes y los límites de su pericia, tiene confianza en sí mismo y sabe gestionar su imagen.

Normativamente, las iniciativas sobre reconocimiento de títulos emprendidas en la Comunidad Europea durante los años sesenta y setenta, dieron lugar a la especificación, en 1983, de cinco niveles de cualificación profesional, que han sido recientemente refrendados en nuestro país por el Real Decreto 1128/2003 que regula el Catálogo Nacional de Cualificaciones Profesionales ${ }^{6}$. Una propuesta de inserción de los tres niveles anteriores de adquisición de competencias en esta estructura normativa podría ser la siguiente (ver Tabla 3):

Nivel 1 Competencia en un conjunto reducido de actividades de trabajo relativamente simples correspondientes a procesos normalizados, siendo los conocimientos teóricos y las capacidades prácticas a aplicar limitados (nivel de principiante).

Nivel 2 Competencia en un conjunto de actividades profesionales bien determinadas con la capacidad de utilizar los instrumentos y técnicas propias, que concierne principalmente a un trabajo de ejecución que puede ser autónomo en el límite de dichas técnicas. Requiere 
conocimientos de los fundamentos técnicos y científicos de su actividad y capacidades de comprensión y aplicación del proceso (nivel inicial de maestría profesional).

Nivel 3 Competencia en un conjunto de actividades profesionales que requieren el dominio de diversas técnicas y puede ser ejecutado de forma autónoma, comporta responsabilidad de coordinación y supervisión de trabajo técnico y especializado. Exige la comprensión de los fundamentos técnicos y científicos de las actividades y la evaluación de los factores del proceso y de sus repercusiones económicas (nivel consolidado de maestría profesional).

Nivel 4 Competencia en un amplio conjunto de actividades profesionales complejas realizadas en una gran variedad de contextos que requieren conjugar variables de tipo técnico, científico, económico u organizativo para planificar acciones, definir o desarrollar proyectos, procesos, productos o servicios (nivel inicial de experto).

Nivel 5 Competencia en un amplio conjunto de actividades profesionales de gran complejidad realizadas en diversos contextos, a menudo impredecibles, que implica planificar acciones o idear productos, procesos o servicios. Gran autonomía personal. Responsabilidad frecuente en la asignación de recursos, en el análisis, diagnóstico, diseño, planificación, ejecución y evaluación (nivel consolidado de experto).

Tabla 3. Niveles de cualificación profesional de la Un ión Europea.

La adscripción de niveles de competencia y cualificación sigue siendo, no obstante, uno de los aspectos polémicos en la adaptación de las nuevas titulaciones al Espacio Europeo de Educación Superior, como demuestran las dificultades de aprobación y debate suscitado por la propuesta de la nueva Directiva sobre reconocimiento de cualificaciones profesionales que se viene tramitando en las instituciones de la Unión Europea desde 2002 y que ha provocado la movilización de algunos sectores profesionales en nuestro país ${ }^{7}$. Ya un informe sobre los sistemas de cualificación profesional en Europa, preparado para CEDEFOP (Centro Europeo para el Desarrollo de la Formación Profesional) al inicio del proceso de Bolonia, ponía de manifiesto ciertas dificultades de nuestro sistema para integrar dentro de esa estructura de cinco niveles los estudios universitarios, especialmente los de primer ciclo, debido a su situación ambigua entre los niveles 4 y 5 . Con alcance general, el informe llamaba la atención también sobre ciertas lagunas en el esquema de grados de Bolonia, al no haber previsto la acomodación de algunos estudios "pre-grado" de corta duración (Heitmann, $2001)^{8}$.

\section{Esquema general para el diseño de programas basados en competencias}

Para terminar, trazaremos la estructura general del diseño de programas basados en competencias, a partir de la adaptación de un esquema planteado por Le Boterf (2001, p.269) que se especifica en el Gráfico 1 en le página siguiente.

Si integramos el esquema anterior con una propuesta de Martínez Mut (2002), podemos identificar los momentos principales del proceso en las siguientes cuatro fases:

- Política formativa. Está constituida por el conjunto de elementos que fundamentan y dan sentido y orientación al programa de formación. Una 


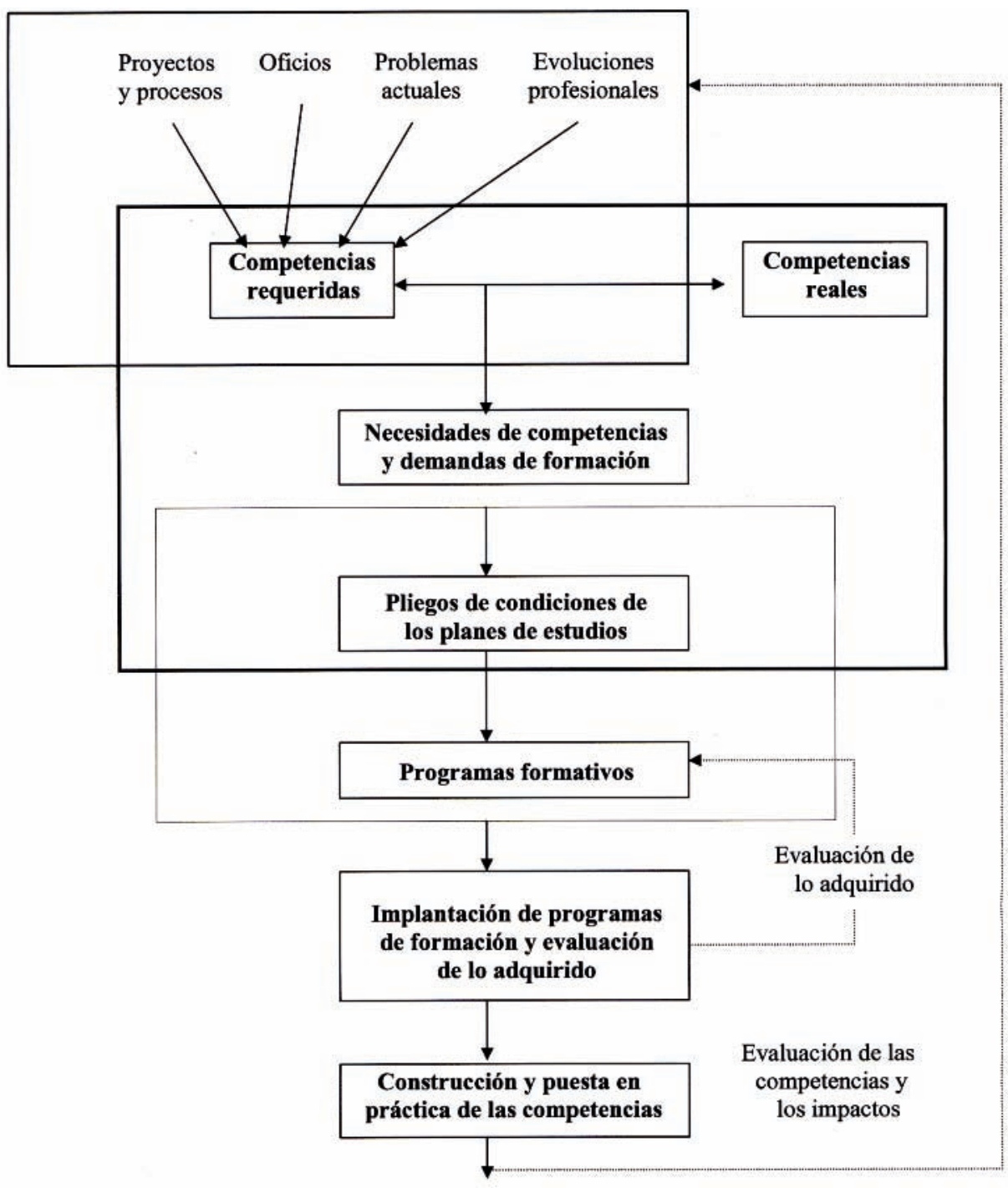

Gráfico 1. Esquema general para el diseño de programas basados en competencias

concreción de las decisiones de esta fase es la definición de prioridades a partir de la detección de necesidades y demandas de formación. No deben de diseñarse programas de formación desvinculados de las necesidades de los sujetos, clientes y gestores de su propio proceso formativo.

- Ingeniería o diseño de la formación. Es el momento de preparación o planificación en el que se establecen las condiciones del programa: objetivos, previsión de recursos personales y materiales, criterios metodológicos, etc. Los programas de 
formación deben considerarse inmersos en un sistema de intervención para satisfacer las necesidades detectadas, en el cual son relevantes las funciones de asesoramiento o tutoría, denominadas también de mentoring o coaching.

- Realización o ejecución del programa. Es el momento pedagógico por excelencia, en el que el alumno cobra especial protagonismo. La ordenación y utilización de los recursos y de las actividades en función de los objetivos y de la realidad de los participantes es una de las condiciones de garantía de la eficacia del programa, así como la adecuada evaluación de los resultados y procesos implicados lo es de las posibilidades de mejora. La evaluación de la eficacia de la formación debe asegurarse utilizando procedimientos de recogida de información que permitan el feed-back y el feed-before constantes del sistema.

- Puesta en práctica y transferencia de lo aprendido. Trata de asegurar que se satisfacen las necesidades que dieron origen al programa y supone el retorno al punto de origen de detección de nuevas necesidades y nuevas demandas. La eficiencia del sistema exige una evaluación a medio y largo plazo del cambio de comportamiento profesional o de la aplicación de las competencias individuales adquiridas, que se realice de manera tan objetiva y científica como la evaluación inmediata de cada actividad.

\section{Reflexión final}

Las competencias constituyen un recurso para orientar los procesos educativos, pero no son la panacea para resolver todos los problemas de la enseñanza superior. Hay que evitar el error de suponer que lo que no se integra en este enfoque no tiene ya cabida en la universidad del futuro. Por el contrario, la transición hacia un futuro desconocido supone una formación para la incertidumbre, la innovación y la imaginación (UNESCO, 1993). En la empresa, lo mismo que en la universidad, un enfoque de formación en competencias actual y realista debe, por ello, completarse con la promoción de algunas actitudes básicas, tales como:

- La experimentación. Cada persona debe situarse en una actitud de investigación y comprobación constante. Con la contrastación de sus teorizaciones en la realidad podrá aprender y generar nuevos campos de exploración.

- El desarrollo personal. Sin identidad individual y cierto grado de autoestima es muy difícil que una persona pueda evolucionar al ritmo de las rápidas transformaciones sin que ello afecte a su equilibrio personal y profesional.

- La multiculturalidad. Cada vez más, el alumno que tendremos delante será una persona que trabaja, o trabajará, una parte del tiempo en su país y el resto en otros, con gente de otras nacionalidades y culturas. Necesariamente la formación debe ser intercultural, es decir, debe desarrollar las competencias imprescindibles para compartir experiencias profesionales y crear con otros individuos que tienen unos esquemas y estilos diferentes a los propios.

Ya no basta, por tanto, con adquirir competencias, se debe dar paso a lo que Biolley ya nos adelantaba en 1992 y que el autor denomina formación-exploración: 
La formación-exploración se concibe esencialmente no para transmitir conocimientos, sino para comenzar a aprender la profesión de mañana. Es decir, para desarrollar a la vez un deseo, una percepción de la necesidad de hacerlo y un enfoque individual y colectivo de superación permanente del estado adquirido en un momento dado (p.217).

Sobre todo, el diseño de titulaciones y programas en el EEES no debe perder de vista que, como decíamos al principio, desde la perspectiva de la Unión Europea el proceso de convergencia se enmarca en la evolución hacia una economía y una sociedad basadas en el conocimiento. Una evolución que tiene uno de sus principales ejes en la promoción de políticas de I+D y en la creación del "Espacio Europeo de Investigación" (EEI), que involucra de manera especial a la universidad, según reconoció la Comisión Europea en su comunicación El papel de las universidades en la Europa del conocimiento (2003, p.3):

La Agenda de Lisboa insta a la participación de numerosos sectores, entre los cuales las universidades desempeñan un papel especialmente relevante, debido a su doble misión tradicional de investigación y enseñanza, a su papel cada vez más importante en el complejo proceso de innovación, y a sus demás aportaciones a la competitividad de la economía y a la cohesión social, como por ejemplo, su función en la vida ciudadana y en materia de desarrollo regional.

Este enfoque adquiere hoy un valor añadido dentro de una visión sistemática de la educación comprometida con la formación a lo largo y ancho de toda la vida. En este contexto, los procesos de identificación, análisis y desarrollo de competencias son necesarios si se quieren lograr dos objetivos ambiciosos y no precisamente contrapuestos, esto es, conseguir una educación para todos de calidad e integrar la educación y la formación dentro de un sistema de ciencia, tecnología y gestión del conocimiento. 


\section{Referencias}

Aristóteles. (1999) Metafisica. (Traducción de Francisco Larroyo). México, Porrúa.

Aubrun, S. \& Orifiamma, R. (1990) Les competentes de 3em. dimension. Paris, Conservatorio de Arts e Metiers.

Bárcena, F. (1994) La práctica reflexiva en educación. Madrid, Editorial Complutense.

Barrantes, E., Torres, E., Marín, L.F., Bustamante, G. \& Gómez, J.H. (2001) El concepto de competencia. Bogotá, Sociedad Colombiana de Pedagogía.

Bunk, G.P. (1994) La transmisión de las competencias y la formación y perfeccionamiento profesionales de la RFA. Revista Europea de Formación Profesional, 1, 8-14.

Comisión de las Comunidades Europeas. (2003) El papel de las universidades en la Europa del conocimiento, COM (2003) 58 final.

Communication of the Conference of European Ministers Responsible for Higher Education. (2003) Realising the european higher education area. Berlin, Bologna Process.

Communication of the Conference of European Ministers Responsible for Higher Education. (2005) The european higher education area - achieving the goals. Berlin, Bologna Process.

Consejo Europeo de Lisboa. Conclusiones de la Presidencia. Boletín de la Unión Europea, 3-2000, punto 1.5.5.

Chomsky, N. (1970) Aspectos de la teoría de la sintaxis. Madrid, Aguilar.

Corominas, J. (1987) Breve diccionario etimológico de la lengua castellana. Madrid, Gredos.

Corripio, F. (1984) Diccionario etimológico general de la lengua castellana. Barcelona, Bruguera.

Echeverría, B., Isus, S. \& Sarasola, L. (1999) Formación para el desarrollo de la profesionalidad. Tesalónica, CEDEFOP.

European Comisión. (2005) ECTS Users' guide. European credit transfer and acumulation system and the diploma supplement. Brussels, Directorate-General for Education and Culture.

EURYDICE. (2002) Las competencias clave. Madrid, MECD.

Fernández-Salinero, C. (2004) Un enfoque pedagógico para el diseño de programas de formación en contextos organizativos. Revista Interuniversitaria de Teoría de la Educación, $16,109-144$.

Fueyo, A. (2004) El diseño de la formación basado en competencias en las titulaciones de Pedagogía y Educación Social. Organización y Gestión Educativa, 6, 18-21.

Gallego, M. (2000) Gestión humana basada en competencias. Contribución efectiva al logro de los objetivos organizacionales. Revista Universitaria EAFIT, 119, 63-71.

Gallego, R. (1999) Competencias cognoscitivas. Un enfoque epistemológico, pedagógico y didáctico. Santafé de Bogotá, Cooperativa Editorial Magisterio.

Gardner, H. (1987) Arte, mente y cerebro. Barcelona, Paidós.

Gardner, H. (1993) La mente no escolarizada. Barcelona, Paidós.

Gardner, H. (1988) La nueva ciencia de la mente. Barcelona, Paidós.

González, J. \& Wagenaar, R. (2003) Tuning educational structures in Europe. Informe final. Fase 1. Bilbao, Universidad de Deusto.

González, J. \& Wagenaar, R. (2005) Tuning educational structures in Europe II. Universities contribution to the Bologna Process. Bilbao, Universidad de Deusto.

Grupo de Trabajo B "competencias clave". (2004) Competencias clave para un aprendizaje a lo largo de la vida. Un marco de referencia Europeo. Bruselas, Comisión Europea.

Habermas, J. (1987) Teoría de acción comunicativa. T. I y T. II. Madrid, Taurus.

Heitmann, G. (2001) European structures of qualification levels. Reports on recent developments in Germany, Spain, France, The Netherlands and in the United Kingdom (England \& Wales). Vol. 3. Luxembourg, Office for Official Publications of the European Communities.

Hyland, T. (1994) Competence, education and NVQs dissenting perspectives. London, Cassel, Redwood Books, Trowbridge, Wiltshire.

Hymes, D. (1980) Paraetnografias de la comunicación. Santafé de Bogotá, Universidad Nacional de Colombia. 
Iriarte, M. (2005) Nuevas competencias para vivir la complejidad. Capital Humano, 193, 34-41.

Jurado, F. (2003) El doble sentido del concepto competencia. Magisterio, Educación y Pedagogía, 1, 14-16.

Le Boterf, G. (2001) Ingeniería de las competencias. Barcelona, Gestión 2000.

Levy-Leboyer, C. (1997) Gestión de las competencias. Barcelona, Gestión 2000.

Martínez Mut, B. (2002) Calidad en las organizaciones y formación. En P. Pineda. (Coord.). Pedagogía laboral (pp.173-200). Barcelona, Ariel.

Mertens, L. (2000) La gestión por competencia laboral en la empresa y la formación profesional. Madrid, OEI.

Morín, E. (1996) Introducción al pensamiento complejo. Barcelona, Gedisa.

Nickerson, R., Perkins, D. \& Smith, E. (1994) Enseñar a pensar: Aspectos de la aptitud intelectual. Barcelona, Paidós.

Noguera, J. (2004) Las competencias básicas. Ponencia presentada a la XIX Semana Monográfica de Educación Santillana. Educación de calidad para todos: Iniciativas Iberoamericanas. Madrid, Santillana.

OECD. (2002) Definition and selection of competences: Theoretical and conceptual foundations. OECD/SFSO, DeSeCo Background Paper.

Perrenoud, Ph. (2004) Diez nuevas competencias para enseñar. Barcelona, Grao.

Platón. (1972) Diálogos: La República o el Estado. (Versión, nuevamente revisada, de Patricio Azcárate). Madrid, EDAF.

Pozo, J.I. (Ed.). (1994) La solución de problemas. Madrid, Santillana.

Pozuelos, F.J. \& Romero, A. (2002) Decidir sobre el curriculum: Distribución de competencias y responsabilidades. Sevilla, Morón.

Sánchez Cerezo, S. (Dir.). (1991) Tecnología de la educación. Madrid, Santillana.

Sarramona, J. (2004) Las competencias básicas en la educación obligatoria. Barcelona, Ceac.

Sarramona, J., Domínguez, E., Noguera J. \& Vázquez, G. (2005) Las competencias en la secundaria y su incidencia en el acceso a la universidad. En E. Chapapría (Ed.). El Espacio Europeo de Educción Superior (pp.199-251). Valencia, UPV/ICE.

Spencer, J.R., McClelland, D. \& Spencer, S.M. (1994) Competency. Assesment methods, history and state of the art. Hay/Mc, Research Press.

Tobón, S. (2004) Formación basada en competencias. Bogotá, Ecoe.

Tobón, S. \& Fernández Tobón, J.L. (2002) Modelo pedagógico basado en competencias. Medellín, FUNORIE.

Torrado, C. (2003) Diversigrama de competencias: Lo último en agrupaciones de competencias. Capital Humano, 165, 34-44.

UNESCO. (1993) Relato general de la cumbre de pensamiento "Visión Iberoamericana 2000". Guatemala, UNESCO.

Van Vucht Tijssen, L. \& De Weert, E. (2005) From erudition to academic competence, Revista Española de Pedagogía, 230, 123-146.

Verón, E. (1971) El proceso ideológico. Buenos Aires, Tiempo contemporáneo.

Verón, E. (Ed.). (1969) Lenguaje y comunicación social. Buenos Aires, Nueva Visión. 


\section{Notas}

1 Estos acuerdos fueron posibles gracias a resoluciones y acciones que la antigua Comunidad Económica Europea aprobó y puso en marcha. La resolución del Consejo, de 6 de junio de 1974, relativa al reconocimiento mutuo de títulos, certificados y otros diplomas es un claro ejemplo de estos antecedentes. También lo es la Resolución del Consejo de Ministros de Educación, de 9 de febrero de 1976, sobre un programa de acción en materia de educación, punto de arranque entre otras iniciativas del Programa Erasmus, que llega a concretarse en la Decisión 87/327/CEE del Consejo, de 15 de junio de 1987, que respalda la acción comunitaria en mat53 movilidad de los estudiantes, germen necesario para las actuales decisiones que establecen el EEES. Otras acciones sin duda importantes en este mismo sentido fueron la creación del Instituto Universitario Europeo en 1972 y la Red de Centros Nacionales de Información para el Reconocimiento Académico (NARIC) en 1984.

2 En el campo profesional ser competente supone demostrar la capacidad para desempeñar un trabajo. Así, la Ley Orgánica 5/2002 de 19 de junio, de las Cualificaciones y de la Formación Profesional, define la competencia como "el conjunto de conocimientos y capacidades que permiten el ejercicio de la actividad profesional conforme a las exigencias de la producción y el empleo" (Boletín Oficial del Estado, 20-6-2002).

3 Actualmente, ya se ha publicado la Fase II del Proyecto Tuning, cuyas novedades más sobresalientes consisten en la incorporación de los Estudios Europeos y de Enfermería a los ya analizados en la Fase I, así como en la identificación de las competencias específicas de estos estudios (González y Wagenaar, 2005).

4 El conocimiento tácito es personal, difícil de formalizar y comunicar, y el conocimiento explícito o codificado, es sistemático y transferible a través del lenguaje formal.

5 En el ámbito específicamente escolar se habla de competencias básicas para referirse a aquellas que deberían ser desarrolladas en los niveles de educación obligatoria, enfocadas a la comprensión y resolución de problemas de la vida cotidiana, mediante el trabajo coordinado en los diferentes ámbitos curriculares (Sarramona, 2004 y Noguera, 2004).

6 Boletin Oficial del Estado de 17-9-2003.

7 Información normativa disponible en europa.eu.int/prelex/detail_dossier_real.cfm?CL=es\&DosId=172279

8 El comunicado de Bergen ha introducido una vía de flexibilización que puede resultar significativa en el marco de ciertas discusiones, al prever la posibilidad, "dentro de los contextos nacionales”, de completar el esquema general de tres ciclos (una vez incluido el doctorado) con "cualificaciones intermedias" (Communication of the Conference of European Ministers Responsible for Higher Education, 2005, p.2). 
154 Encounters/Encuentros/Rencontres 